\title{
Detection of Through-Deck Type Fatigue Cracks in Steel Bridges by Self-Reference Lock-in Thermography
}

\author{
Y. Izumi ${ }^{1, a}$, T. Sakagami ${ }^{2}$, S. Kubo ${ }^{1}$ and T. Tamakoshi ${ }^{3}$ \\ ${ }^{1}$ Dept. of Mech. Eng., Graduate School of Eng., Osaka University, 2-1 Yamadaoka, Suita, Osaka, \\ Japan \\ ${ }^{2}$ Dept. of Mech. Eng., Graduate School of Eng., Kobe University, 1-1 Rokkodai, Nada, Kobe, Japan \\ ${ }^{3}$ Bridge and Structures Division, Road Department, National Institute for Land \& Infrastructure \\ Management, 1, Asahi, Tsukuba, Ibaragi, Japan
}

\begin{abstract}
A new remote nondestructive inspection technique, based on thermoelastic temperature measurement by infrared thermography, is developed for detection and evaluation of fatigue cracks propagating from welded joints in steel bridges. Fatigue cracks are detected from localized high thermoelastic temperature change at crack tips due to stress singularity under variable loading from traffics on the bridge. Selfreference lock-in data processing technique is developed for the improvement of signal/noise ratio in the crack detection process. The technique makes it possible to perform correlation processing without an external reference signal. It is very difficult to detect through-deck type fatigue cracks in steel decks by the conventional NDT technique, since they are not open to the inspection. In this paper, self-reference lock-in thermography is applied for detection of through-deck type fatigue cracks. Experiments are carried out to steel deck sample, which simulates an actual steel bridge, during crack propagation test. It is found that significant stress concentration zone can be observed near the crack front, which enabled us to detect through-deck type fatigue cracks and to estimate its size.
\end{abstract}

\section{Introduction}

In recent years, fatigue crack propagations in aged steel bridge which may lead to catastrophic failures of the structures have become a serious problem. For large-scale steel structures such as steel decks in steel bridges, non-destructive inspection of deteriorations and fatigue damages are indispensable for securing their safety and for estimating their remaining strength.

Through-deck type fatigue cracks which initiated from weld root and propagated through deck plate causes serious problems in aging steel bridges [1-2]. Through-deck type fatigue cracks are not open to the inspection surface as shown in Figure 1, therefore they are very difficult to be detected by the conventional nondestructive testing techniques such as visual testing or magnetic particle testing. Only ultrasonic testing has been applied to the detection of the through-deck type cracks. However

\footnotetext{
a e-mail : y-izumi@saos.mech.eng.osaka-u.ac.jp
} 


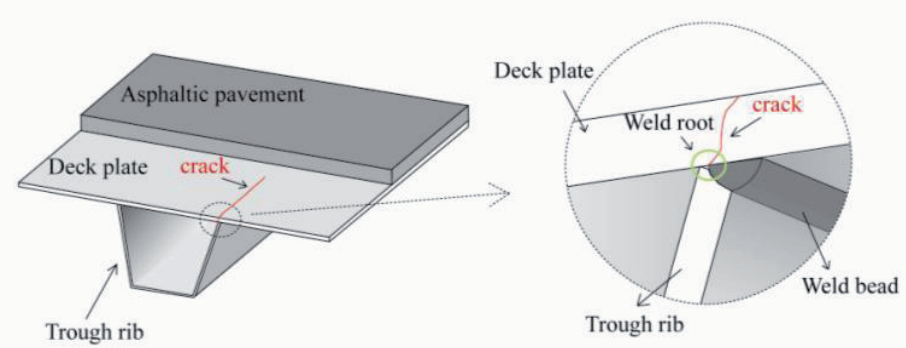

Fig. 1. Illustrations of through-deck type fatigue cracks.

ultrasonic testing is a time- and labor-consuming technique. Development of remote and noncontacting effective nondestructive testing techniques for back surface crack is required.

Infrared thermography is an effective tool for remote and full-field measurement of temperature distribution on the objective surface. Using an infrared thermography, change in sum of the principal stresses can be measured based on thermoelastic temperature changes. Bridges in service are subjected to random variable loading by traffics on the bridge. Thus fatigue cracks can be detected from localized high thermoelastic temperature change observed at crack tips due to stress singularity. The present authors developed a self-reference lock-in thermography [3], which does not require any external reference signals and can be employed even under random loading. Nondestructive inspections of fatigue cracks in steel bridges were conducted by the proposed selfreference lock-in thermography in the previous paper [4].

In this paper, detection of through-deck type fatigue cracks in a steel deck specimen is conducted by the self-reference lock-in thermography. Experiments are carried out for steel deck sample, which simulates an actual steel bridge, during crack propagation test under point load and traveling wheel load.

\section{Thermoelastic stress measurement}

Dynamic stress change causes temperature change under the adiabatic condition in solid. This phenomenon is called as thermoelastic effect and is described by the following Lord Kelvin's equation that relates temperature change $(\Delta T)$ to a change in the sum of the principal stresses $(\Delta \sigma)$ under the cyclic variable loading.

$$
\Delta T=-\frac{\alpha}{\rho C_{p}} T \Delta \sigma=-K_{m} T \Delta \sigma
$$

$\alpha$ : Coefficient of thermal expansion

$\rho$ : Mass density

$C_{p}:$ Specific heat under constant pressure

$T$ : Absolute temperature

A change in the sum of the principal stress $(\Delta \sigma)$ is obtained by measuring temperature change $(\Delta T)$ ) using the infrared thermography. Usually, $\Delta T$ is quiet small. In cases like this, lock-in processing for noise reduction is needed.

\section{Self-reference lock-in thermography}

The self-reference lock-in thermography [3], developed by the present authors, enabled us to measure the distribution of relative intensity of applied stress under random loading without using any external load signal. In the self-reference lock-in thermography, a reference signal was 
constructed from the reference region arbitrarily set on the same sequential infrared images on thermoelastic temperature change. Distribution of relative intensity values of the thermoelastic temperature change against that in the reference region can be obtained by the following least squares approach even under the random loading, provided that the temperature change in the reference region has the similar and in-phase waveform as the objective area of the measurement.

Assume that a body is subjected to a random load whose waveform is expressed as $f_{n}$. The infrared signal in an objective region can be approximated as follows.

$$
Y_{n}=a+b f_{n}
$$

Where $a$ is the DC offset, $b$ is an influence coefficient of the reference, $n$ is the frame number. To calculate the influence coefficient, the square sum of deviations between $Y_{n}$ and infrared signal $y_{n}$ obtained from the region defined by the following equation is minimized.

$$
\Delta^{2}=\sum_{n=1}^{N}\left(y_{n}-Y_{n}\right)^{2}
$$

Where $N$ is the total frame number. Then, $b$ is obtained by the following equation.

$$
b=\frac{\left|\begin{array}{cc}
N & \Sigma y_{n} \\
\Sigma f_{n} & \Sigma y_{n} f_{n}
\end{array}\right|}{\left|\begin{array}{cc}
N & \Sigma f_{n} \\
\Sigma f_{n} & \Sigma\left(f_{n}\right)^{2}
\end{array}\right|}=\frac{N \Sigma y_{n} f_{n}-\Sigma y_{n} \Sigma f_{n}}{N \Sigma\left(f_{n}\right)^{2}-\left(\Sigma f_{n}\right)^{2}}
$$

When this calculation is performed on all the pixels of infrared thermography, it is possible to obtain the correlation between the infrared signal in reference region and that in any region. Values of $b$ indicate a relative intensity of thermoelastic temperature change against that in the reference region. The self-reference lock-in method does not require any external reference signals and can be applied even under the random loading. Obtained values of $b$ are effectively employed for detection of stress concentration area around notches or cracks.

\section{Crack propagation test under point loading}

\subsection{Experimental procedure}

Detection of through-deck type fatigue cracks in a steel deck specimen was conducted by the selfreference lock-in thermography. The steel deck sample, which simulated a part of the actual steel deck of the bridge, is shown in Figure 2. The thickness of the deck plate was $19 \mathrm{~mm}$. Dimensions of cross section of the trough rib were indicated as U320 $\times 240 \times 6$; i.e., top width, height and thickness were $320 \mathrm{~mm}, 240 \mathrm{~mm}$ and $6 \mathrm{~mm}$, respectively. In order to initiate and propagate a fatigue crack, cyclic load was applied to the specimen using a servo-hydraulic testing machine. Cyclic load was applied at two points through $200 \mathrm{~mm} \times 200 \mathrm{~mm} \times 15 \mathrm{~mm}$ solid rubbers shown in Figure 2, which simulated single tires. Loading positions are set at the centers of trough ribs on the deck plate. Loading frequency was $9 \mathrm{~Hz}$, and minimum load and maximum load was $-10 \mathrm{kN}$ and $-110 \mathrm{kN}$, respectively.

Visible image of the infrared measurement area and schematic illustration of cross-section in the infrared measurement are shown in Figure 3 and Figure 4, respectively. Thermoelastic stress measurement area is set near intersection of deck plate, trough rib and cross rib at the part " $\mathrm{A}$ " in Figure 2, where crack initiation and propagation is expected. A fatigue crack was initiated from the 


\section{EPJ Web of Conferences}

back surface of the weld bead shown in Figure 4(a), and propagated to the deck plate and longer direction of a trough rib indicated in Figure 4(b); however it was not open to the inspection surface. Infrared measurement is conducted during fatigue propagation test on regular interval. The change in a stress distribution in the measurement area by the crack initiation and propagation was observed. The temperature change under cyclic loading was measured by the IR camera with MCT array detector. Infrared measurement frequency was set to $113 \mathrm{~Hz}$ and obtained sequential infrared data were processed by self-reference lock-in technique.
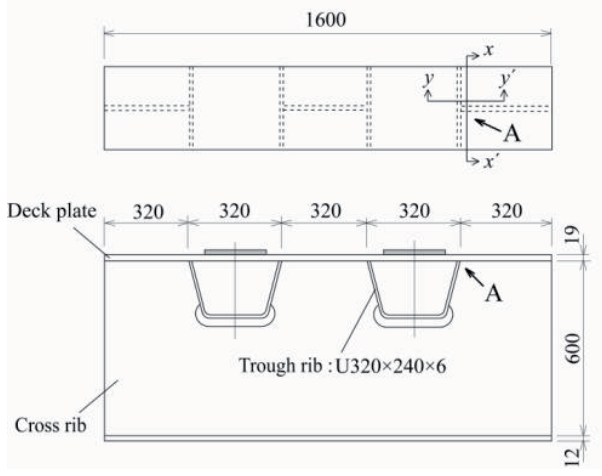

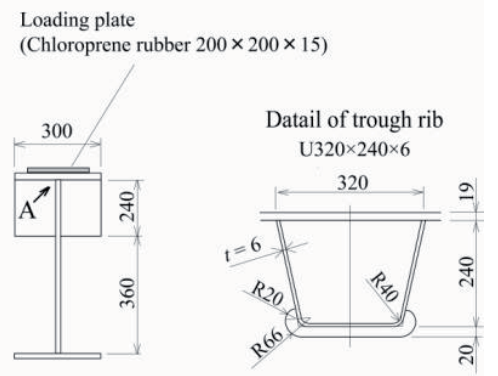

Fig. 2. Configuration of steel deck sample.

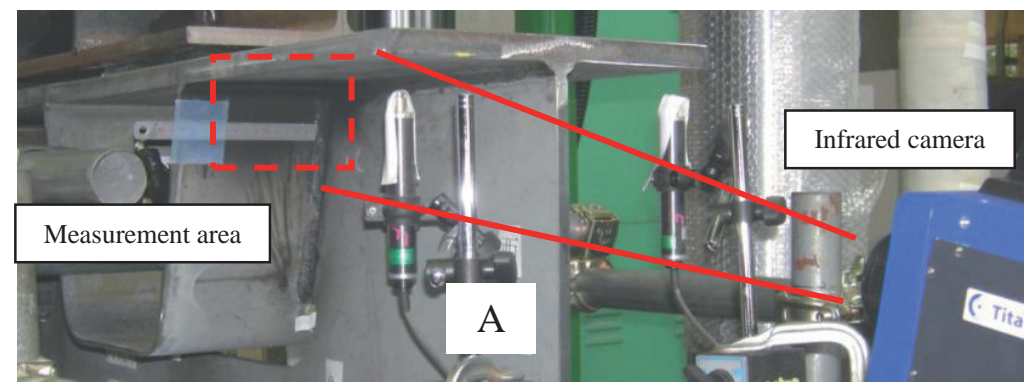

Fig. 3. Infrared measurement area for through-deck type fatigue cracks.

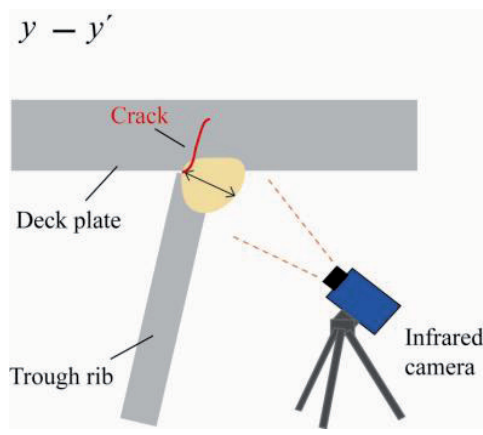

(a)

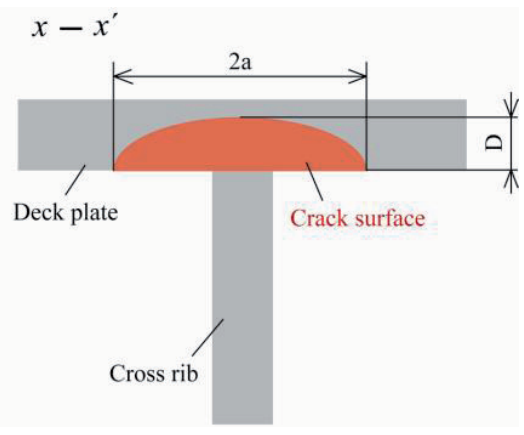

(b)

Fig. 4. Schematic illustration of cross-section in the infrared measurement. (a) Cross-section alone the line y-y' shown in Figure 2. (b) Cross-section alone the line $x-x$ ' shown in Figure 2. 


\subsection{Experimental results}

The experimental results obtained by the self-reference lock-in thermography in crack propagation process, are shown in Figure 5. The contrast shows the relative intensity values of the thermoelastic temperature change against that in the reference region "Ref" in the figure.

Characteristic stress concentration in the deck-to-rib weld bead was not shown in the early stage of fatigue testing as shown in Figure 5(a). On the other hand, significant stress concentration zones at the location away from the intersection of the cross rib in the weld bead are found in Figures 5(b), (c) and (d). It is also found that this stress concentration zone moved away from cross rib with the increasing loading cycles.

Experimental results of crack measurement between the self-reference lock-in thermography and the ultrasonic inspection are compared in Table 1. In this table, distance between center of stress concentration zone and cross rib corresponds to the half crack length obtained by ultrasonic inspection. It was found that significant stress concentration zone appeared near the semi-elliptical crack front, which enabled us to detect through-deck type fatigue cracks and to estimate its half crack length in longer direction of trough rib.

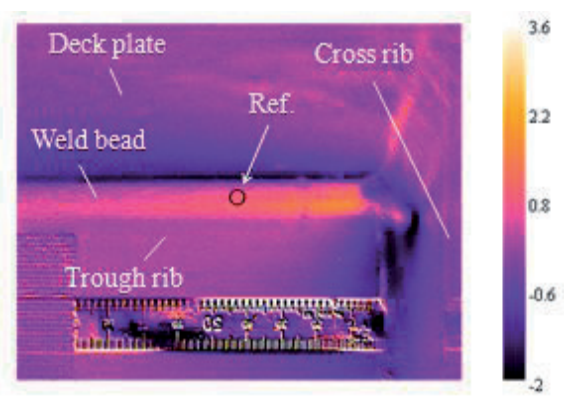

(a)

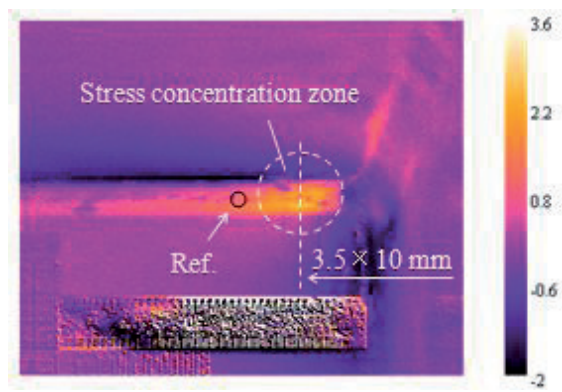

(c)

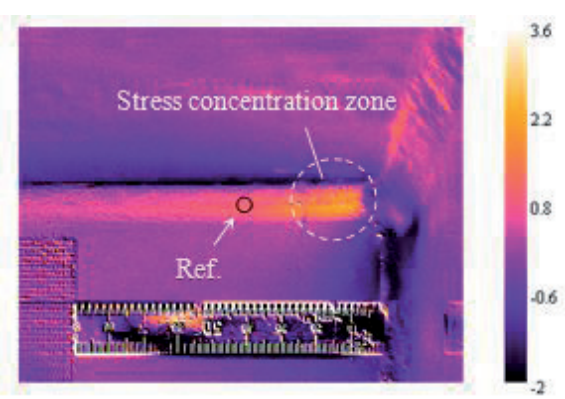

(b)

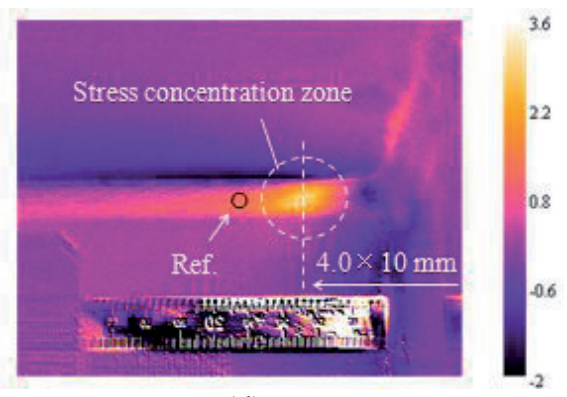

(d)

Fig. 5. Results of self-reference lock-in thermography for detection of through-deck type fatigue cracks in crack propagation test under static load. (a) Number of load cycles, $4.0 \times 10^{4}$. (b) Number of load cycles, $5.0 \times 10^{5}$. (c) Number of load cycles, $1.0 \times 10^{6}$. (d) Number of load cycles, $2.0 \times 10^{6}$.

Table 1. Comparison of experimental results of crack measurement between the self-reference lock-in thermography and the ultrasonic inspection.

\begin{tabular}{c|c|c}
\hline \hline $\begin{array}{c}\text { Number of } \\
\text { load cycles }\end{array}$ & $\begin{array}{c}\text { Distance from cross rib to stress concentration } \\
\text { zone obtained by the self-reference lock-in } \\
\text { thermography }\end{array}$ & $\begin{array}{c}\text { Half crack length obtained by } \\
\text { the ultrasonic inspection }\end{array}$ \\
\hline \hline $5.0 \times 10^{5}$ & (Difficult to determine) & $1.7 \times 10 \mathrm{~mm}$ \\
\hline $1.0 \times 10^{6}$ & $3.5 \times 10 \mathrm{~mm}$ & $3.4 \times 10 \mathrm{~mm}$ \\
\hline $2.0 \times 10^{6}$ & $4.0 \times 10 \mathrm{~mm}$ & $4.0 \times 10 \mathrm{~mm}$ \\
\hline \hline
\end{tabular}




\section{Crack propagation test under the traveling wheel load}

\subsection{Experimental procedure}

Detection of through-deck type fatigue cracks in a steel deck sample under the traveling wheel load was conducted by the self-reference lock-in thermography. The steel deck test sample simulated a part of the actual steel deck of the bridge. Illustration of steel deck sample is shown in Figure 6. Dimensions of the sample were $3,880 \mathrm{~mm}$ in length, 2,810 $\mathrm{mm}$ in width and $953 \mathrm{~mm}$ in height. The thickness of the deck plate was $12 \mathrm{~mm}$. Dimensions of cross section of the trough rib were indicated as U320×240×6; i.e., top width, height and thickness were $320 \mathrm{~mm}, 240 \mathrm{~mm}$ and $6 \mathrm{~mm}$, respectively. Schematic illustration of fatigue testing apparatus of traveling wheel loading is shown in Figure 7. Frequency and stroke of the traveling wheel was $0.5 \mathrm{~Hz}$ and $2 \mathrm{~m}$, respectively. The load was set to be $120 \mathrm{kN}$. Steel loading plate was laid between the deck plate of the sample and traveling wheel as shown in Figure 6, so that wheel load was separately applied to the deck via this loading plate.

Thermoelastic stress measurement area was set near intersection of deck plate, trough rib and cross rib, where crack initiation and propagation is expected. Visible image of the measurement area by the infrared thermography is shown in Figure 8. A fatigue crack was initiated from the back surface of the weld bead shown in Figure 4(a), and propagated to the deck plate and longer direction of a trough rib indicated in Figure 4(b).

Infrared measurement is conducted during fatigue propagation test on regular interval. The change in a stress distribution in the measurement area by the crack initiation and propagation was observed. The temperature change under traveling wheel loading was measured by the IR camera with MCT array detector. Infrared measurement frequency was set to $113 \mathrm{~Hz}$ and obtained sequential infrared data were processed by self-reference lock-in technique.

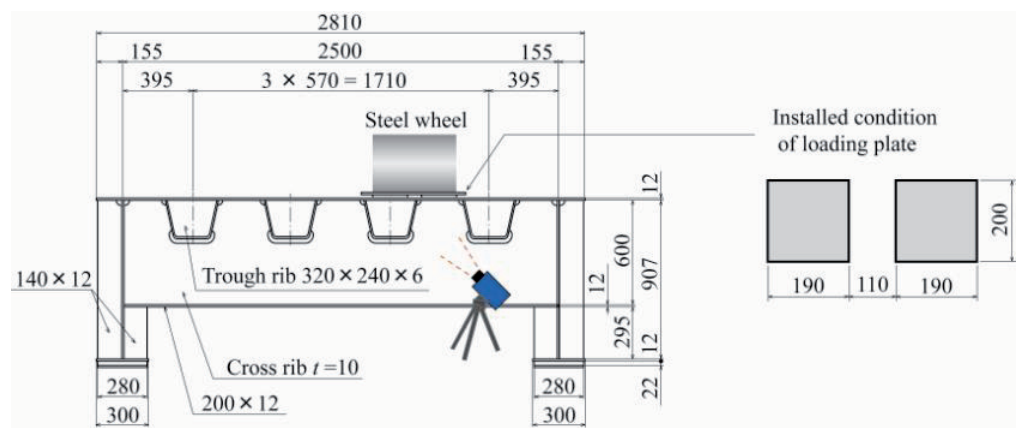

Fig. 6. Configurations of steel deck sample for traveling wheel loading test (mm).

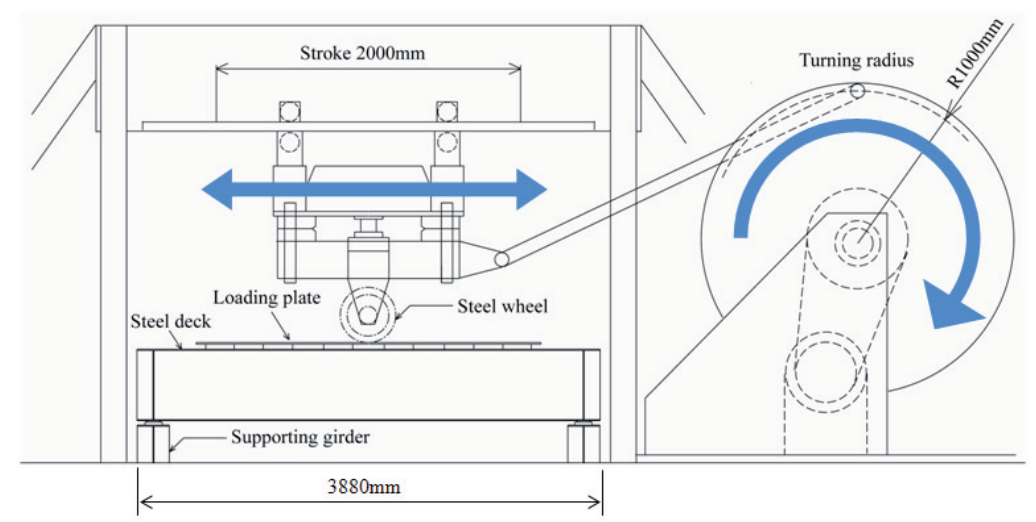

Fig. 7. Testing apparatus of traveling wheel loading. 


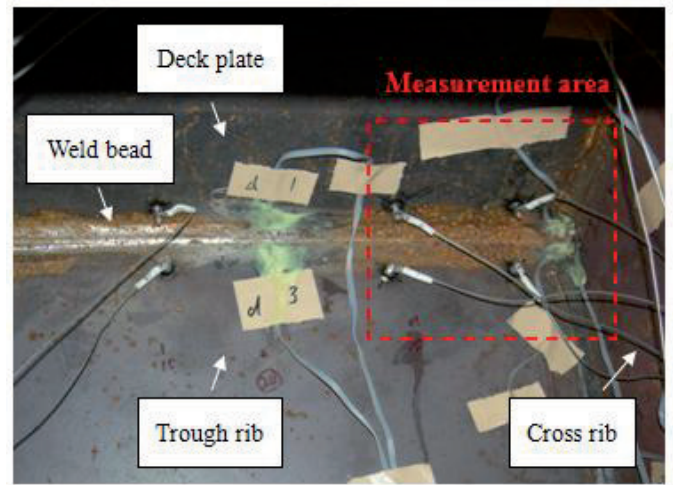

Fig. 8. Visible image of the infrared measurement area.

\subsection{Experimental results}

The experimental results obtained by the self-reference lock-in thermography in crack propagation process, are shown in Figure 9. The contrast shows the relative intensity values of the thermoelastic temperature change against that in the reference region "Ref" in the figures.

Characteristic stress concentration in the deck-to-rib weld bead was not shown in the early stage of fatigue testing as shown in Figure 9(a). On the other hand, significant stress concentration zone in the weld bead were found in Figures 9(b), (c) and (d). It is also found that this stress concentration zone moved away from cross rib with the increasing loading cycles. The distance between the center of stress concentration zone and the cross rib was measured and shown in the figure.

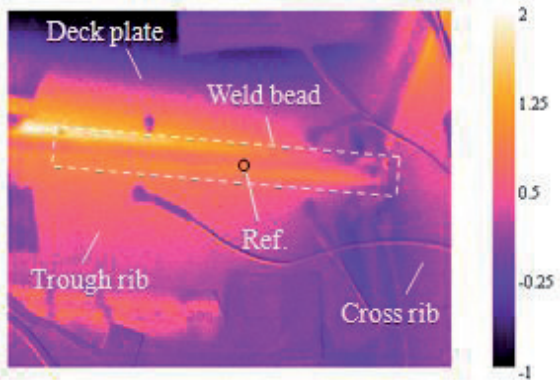

(a)

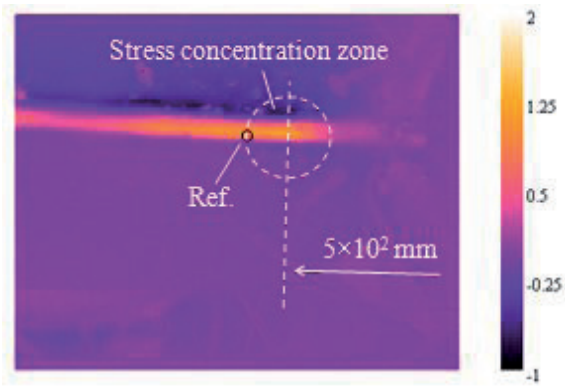

(c)

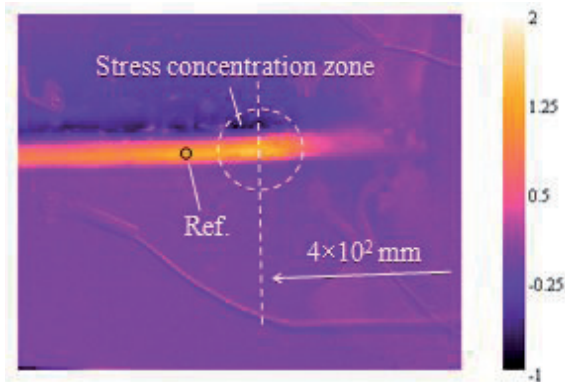

(b)

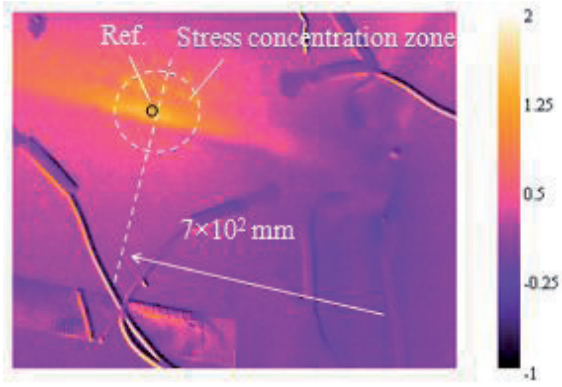

(d)

Fig. 9. Results of self-reference lock-in thermography for detection of through-deck type fatigue crack under traveling wheel loading. (a) Number of load cycles, $8.0 \times 10^{2}$. (b) Number of load cycles, $1.63 \times 10^{6}$.

(c) Number of load cycles, $2.22 \times 10^{6}$. (d) Number of load cycles, $2.62 \times 10^{6}$. 
After 2,620,000 cycles of wheel loading, surface crack length on the deck plate was measured by the magnetic-particle test. The location of the crack tip can be estimated around $80-90 \mathrm{~mm}$ from cross rib. The location of the crack tip on the deck plate largely coincides with that of the stress concentration zone in the self-reference lock-in result. It is found that significant stress concentration zone can be observed near the crack front, which enabled us to detect through-deck type fatigue cracks under traveling wheel loading condition.

\section{Conclusions}

Detection of through-deck type fatigue cracks in a steel deck was conducted by the self-reference lock-in thermography. Experiments were conducted in crack propagation test under point load and traveling wheel load. In both experiments, it was found that significant stress concentration zone can be observed near the semielliptical crack front, which enabled us to detect through-deck type fatigue cracks and to estimate its half crack length in longer direction of trough rib.

\section{Acknowledgement}

This research was partly supported by Japan Society for the Promotion of Science through the Grantin Aid for Scientific Research, and by National Institute for Land Infrastructure Management through the Research Grant.

\section{References}

1. Y. Horie, Y. Takada, Proceedings of The 10th Symposium on Steel Structure and Bridge, Japan Society of Civil Engineers, pp.55-69, (2007)

2. Y. Hirabayashi, H. Ushikoshi, T. Kinomoto, Proceedings of The 10th Symposium on Steel Structure and Bridge, Japan Society of Civil Engineers, pp.39-53, (2007)

3. T. Sakagami, T. Nishimura, S. Kubo, Y. Sakino, K. Ishio, Transactions of the Japan Society of Mechanical Engineers. A, 73, 724, pp.1860-1867, (2006)

4. Y. Izumi, T. Sakagami, S. Kubo, T. Tamakoshi, Proceeding of ASCE 2008 International Orthotropic Bridge Conference, pp.502-513 (CD-ROM), (2008) 